\title{
Social Innovation Studies, un paso adelante en la construcción del campo de la innovación social.
}

\section{Social Innovation Studies, a step forward in the construction of the social innovation field.}

\author{
Luz Andrea Cote' 1 - Emilio Ricci' \\ 1 Universidad Católica del Norte, Antofagasta, Chile. \\ sisjournal@ucn.cl@ericci@ucn.cl
}

\section{Resumen:}

\section{(c) (1)}

Los estudios teóricos y bibliométricos realizados sobre la innovación social (IS) indican con frecuencia que, pese al protagonismo que ha ganado en las estrategias de organismos multilaterales y en las estructuras gubernamentales y las políticas públicas de una diversidad de países, el campo adolece de claridad conceptual e investigativa. La falta de revistas especializadas y redes de coautoría habrían incidido en esta situación. A nivel internacional, el enfoque de la IS ha sido marcado principalmente por Europa y Norteamérica, regiones en las que la investigación y la publicación científica se han visto estimuladas por el ascenso del término en programas de desarrollo. No obstante, en Latinoamérica, la IS ha tenido sus propias características y merece un tratamiento con marcos conceptuales pertinentes. Social Innovation Studies (SIS) busca reunir la atomizada publicación latinoamericana en la materia y convocar a los autores de la región para entablar un diálogo internacional basado en un análisis de la realidad regional que aborde un amplio espectro, desde enfoques micro sustentados en análisis de casos, hasta enfoques macro que relacionen la IS con los grandes procesos de cambio social.

Palabras clave: procesos sociales; crisis; América Latina; innovadores sociales; desafíos sociales.

\begin{abstract}
:
Theoretical and bibliometric studies carried out on social innovation (SI) frequently indicate that, despite the prominence that it has been gaining in the strategies of multilateral organizations and in the government structures and public policies of a variety of countries, the field lacks conceptual and investigative clarity. The absence of specialized journals and co-authorship networks has influenced this situation. Internationally, the focus of SI has been guided mainly by Europe and North America, regions where research and scientific publication have been stimulated by the rise of the term in development programs. However, in Latin America, SI has had its own characteristics and deserves a treatment with appropriate conceptual frameworks. Social Innovation Studies (SIS) wants to bring together the atomized Latin American publication on the subject and convene authors from the region to engage in an international dialogue based on an analysis of the regional reality that addresses a wide spectrum, from micro-approaches supported by case analysis to macro-approaches that link SI with great processes of social change.

Keywords: social processes; crisis; Latin America; social innovators; social challenges.
\end{abstract}

Enviado: 2020-09-26 | Aceptado: 2021-08-18 


\section{Introducción}

Si bien el concepto actual de innovación social (IS) puede rastrearse hasta inicios del siglo XX, y si se quiere, se puede asociar a conceptos precedentes de los siglos XVIII y XIX (Jessop, Moulaert, Hulgård \& Hamdouch, 2013; Moulaert, MacCallum \& Hillier, 2013), ha venido a ganar su popularidad actual en los últimos 20 años, desde inicios del siglo XXI (Buckland \& Murillo, 2014; Hernández-Ascanio, Tirado \& Ariza-Montes, 2016; Moulaert et al., 2013). Esto ha sido resultado de la adopción del término por parte de organismos multilaterales y gobiernos de distintos estados -primeramente, de Europa y Estados Unidos- que lo han convertido en una línea estratégica en el diseño de sus políticas. Como se verá, esta posición estratégica de la IS ha suscitado un creciente interés en ella en el campo académico y científico, lo cual ha conducido a la proliferación de publicaciones al respecto (Pacheco et al., 2018; Weerakoon et al., 2016).

Pese a lo anterior, tal y como señalan diversos estudios bibliográficos, revisiones de literatura y trabajos sobre el estado de arte de la investigación y producción teórica sobre IS, el término es aún ambiguo y este tipo de innovación, como campo de estudio, adolece de claridad conceptual y, por tanto, investigativa (Buckland \& Murillo, 2014; Butzin et al., 2014; Jessop et al., 2013; Özbağ et al., 2019; Pacheco et al., 2018). Algunos autores cuentan entre los principales factores para esta falta de claridad la ausencia de revistas científicas especializadas y la falta de redes de coautoría (Özbağ et al., 2019; Weerakoon et al., 2016).

La sumatoria de estos factores, es decir, el crecimiento exponencial del uso del término, acompañado de una percibida vaguedad conceptual, son razones suficientes para impulsar una publicación científica especializada en la materia que ayude a conducir el campo de la IS hacia un acervo teórico y metodológico que de frutos para todos: académicos, gestores de política pública, ONG, empresarios y emprendedores, líderes comunitarios, etc., con especial interés en los innovadores sociales. Sin embargo, a estos motivos se suma uno específico para Latinoamérica: la teoría sobre IS ha sido construida principalmente entre Europa y Estados Unidos, mientras que la región ha sido proveedora de casos de estudio (Hetland \& Evans, 2016).

Social Innovation Studies (SIS) ha decidido crear un espacio de convergencia para los interesados en consolidar la IS como campo de estudio, con el especial ánimo de alentar y encausar la discusión académica en Latinoamérica, región que, aunque tiene mucho que aportar desde su experiencia en IS, no se ha expresado a cabalidad en el ámbito de las publicaciones académicas y científicas. Un objetivo específico es el de facilitar la creación de las mencionadas redes de coautoría en el subcontinente, y, desde allí, favorecer un diálogo internacional más horizontal con investigadores internacionales. No obstante, también lo es el facilitar la emergencia de un foro regional en el que se encuentren investigadores, representantes de los sectores público y empresarial, e innovadores sociales. 
Pero existe una motivación más: como se profundizará en el siguiente apartado, tanto las innovaciones sociales en sí, como el actual auge del concepto, tienen mucho que ver con las crisis vividas tanto en el norte como en el sur global: económicas y financieras, ambientales, sociales, de gobernabilidad, etc. Pues bien, estamos ante una nueva crisis mundial debido a la pandemia por Covid 19 y a todas las medidas que ha sido necesario tomar para mitigar su impacto. Se hace, entonces, imperativo, saber qué está sucediendo en cuanto a respuestas de la sociedad a los distintos aspectos de la emergencia sanitaria y la crisis económica y social, ya que la realidad corre más rápido que la investigación, la teorización y la construcción de políticas públicas. Es el momento de refrescar la discusión sobre la IS con preguntas como: ¿Cuáles de las iniciativas sociales que están dando respuestas a los desafíos de la pandemia pueden entenderse como IS y por qué? O, visto de otro modo, ¿puede ser la IS una alternativa para dar respuestas a las múltiples problemáticas que derivan de la pandemia?, y si es así, ¿cuáles deberían ser los mecanismos e instrumentos para ser fomentada, reproducida y/o escalada? ¿es esto verdaderamente posible? ¿estamos, en realidad, ante un concepto útil para la construcción de conocimiento y el establecimiento de lineamientos para fomentar un mayor bienestar social?

SIS es una invitación a abordar estas y muchas más preguntas desde el conocimiento ya acumulado, pero con apertura a los nuevos interrogantes y a las nuevas respuestas que ya nos está dejando la actual situación global. A continuación desglosamos algunos aspectos que nos parecen relevantes a tener en cuenta antes de plantearnos otras posibles preguntas y temas a abordar en el amplio campo de la IS.

\section{Innovación social, una idea en expansión}

Si bien aún no existe acuerdo sobre quién acuñó el concepto de IS y cuándo sucedió, y aunque las primeras publicaciones científicas que emplean el término se remontan a las décadas de los años sesenta y setenta, una concatenación de factores llevó a que, ya iniciado el siglo XXI, gobiernos, organizamos internacionales, ONG, investigadores, y un gran número de instituciones públicas y privadas lo retomaran y apropiaran, moldeando, a la vez, sus acepciones actuales, y revelando en ellas las preocupaciones y valores contemporáneos.

Diversos autores se han ocupado de señalar los factores que han llevado a la popularidad del concepto de IS, los cuales configuraron en el último cambio de siglo lo que Westley \& Antadze (2010), citando a Carpenter et al. (2009), calificaron como una "tormenta perfecta" (p. 5). El primer factor, y que

\footnotetext{
${ }^{1}$ De acuerdo con Castro-Arroyave \& Duque-Paz (2020), McGowan \& Westley (2017) adjudican la acuñación del término a Lester Fran Wald (sic), aunque en la fuente que citan no figura tal información. Entre tanto, Moulaert, MacCallum \& Hillier (2013), resaltan que no está claro quién utilizó por primera vez la expresión "innovación social", pues, aunque algunos autores la adjudican a James Taylor (1970) en su análisis de la dinámica del desarrollo comunitario en Topeka, Kansas, ellos consideran que el término fue acuñado en el continente europeo, en medio de las revueltas sociales de finales de los años sesenta.
} 
actúa como una especie de paraguas para otros varios, es el de las crisis económicas y financieras que han tenido lugar en el transcurso del siglo XXI, especialmente en Estados Unidos y Europa, y que en esta última llevaron al debilitamiento del estado de bienestar debido a la reducción de recursos asignados para proveer servicios sociales hasta entonces priorizados (Arenilla, 2017; Consiglio \& Zabatino, 2015; Hernández-Ascanio et al., 2016; Parada, Ganga \& Rivera, 2017).

A la pérdida de los beneficios sociales se sumaron otros factores como el decrecimiento o la precarización del empleo, y la acentuación de la desigualdad y de la exclusión social (Consiglio \& Zabatino, 2015; Parada et al., 2017). Asimismo, la escasez de alimentos, relacionada a su vez con el calentamiento global (Westley \& Antadze, 2010), uno de los principales motivadores de innovaciones sociales en todo el mundo. Se agregan a ello los desastres naturales que han dejado numerosas víctimas en lo que va corrido del siglo, el crecimiento de enfermedades discapacitantes de larga duración, las enfermedades crónicas, los problemas de comportamiento crecientes -obesidad, sedentearismo, etc.-, y, por supuesto, las epidemias (Consiglio \& Zabatino, 2015). A los anteriores, Mulgan (como se citó en Consiglio \& Zabatino, 2015) agregó factores que, aunque no representan problemáticas en sí, plantean desafíos motivadores de innovaciones sociales, tales como el alargamiento de la vida media -que repercute en la organización de los sistemas de pensiones, en materia de vivienda y servicios sociales-, y el crecimiento de la diversidad cultural en ciudades y pueblos -que plantea retos para los sistemas de formación y la convivencia, entre otros-. Por último, Westley \& Antadze (2010) señalan un factor que funciona como paraguas, y es la dificultad de comprender la dinámica real de estos problemas debido a su complejidad.

La conjugación de todos estos fenómenos ha llevado a la percepción de insuficiencia de los Estados para dar solución, desde sus estructuras tradicionales y viejos paradigmas, a las problemáticas socioambientales contemporáneas, y para integrar en la acción pública a la sociedad (Arenilla, 2017; Consiglio \& Zabatino, 2015; Murray, Caulier-Grice \& Mulgan, 2010; Parada et al., 2017). Desde la década de los noventa del siglo pasado el Estado había implementado un enfoque eficientista para proveer los bienes y servicios públicos delegando muchas de las funciones públicas a actores no estatales mediante fórmulas como la privatización, desregulación, subcontratación, externalización o la concertación de servicios (Arenilla, 2017). Esto produjo una mayor participación de las organizaciones sin fines de lucro y las empresas privadas en la satisfacción de las necesidades ciudadanas; sin embargo, la falta de incentivos, las fallas en los modelos, y el enfoque en la reducción de costos por parte de la empresa privada, así como la falta de capital, habilidades y recursos de la sociedad civil para llevar a escala las ideas prometedoras, limitaron los impactos de estas innovaciones (Arenilla, 2017; Murray et al., 2010).

Estas debilidades abrieron paso a nuevas formas de entender las relaciones entre Estado, mercado y sociedad con enfoques como el del Estado neoweberiano, la gobernanza, las redes de política pública y el Nuevo Servicio Público, entre otros, que hacen que la idea de lo público ya no 
pueda limitarse a lo administrativo o estatal (Arenilla, 2017). La evolución de estas ideas, sumada a factores como el creciente desarrollo y expansión tecnológicos, el cuestionamiento a la repartición de beneficios que logra la innovación tradicional -centrada en la innovación tecnológica-, un cambio de paradigma hacia una innovación de carácter social (Butzin et al., 2014), la creciente importancia de formas alternativas de economía -civil, del bien común, social, solidaria, colaborativa, azul, circular, etc., y la creciente confianza de las personas en su talento y su disposición para colaborar (Consiglio y Zabatino, 2015), facilitaron la reapropiación del concepto de IS, orientándolo, en términos generales, al "establecimiento de nuevos modelos colaborativos y participativos" que "tratan de conectar el talento y el conocimiento para transformar la acción pública y solucionar los problemas sociales de forma sostenible" (Arenilla, 2017, p. 41).

Como resultado, desde la década de los ochenta se vienen creando institutos y centros dedicados a la investigación y promoción de la IS, así como programas para su fortalecimiento. Se pueden destacar el Centre de Recherche sur les Innovations Cociales (CRISES), de Canadá; el Viennabased Zentrum für Soziale Innovation (ZSI); y, en el Reino Unido, The Young Fundation y National Endowment for Science, Technology and the Arts (NESTA). Los dos últimos han sido los asesores principales en la materia para la Comisión Europea, cuya definición de IS ha tenido gran influencia a nivel mundial, incluida Latinoamérica. ${ }^{2}$ En 2010, la Comisión puso en marcha el Programa Marco de Investigación e Innovación de la Unión Europea, que ve la IS como una de las medidas prioritarias para asumir los principales desafíos de la sociedad europea en materias como salud, energía, seguridad alimentaria, acción por el clima, inclusión y seguridad (Comisión Europea, 2014).

La OCDE, por su parte, desarrolló el LEED Programme (Local Economic and Employment Development), el cual incluye un foro que ha trabajado en la investigación y en la difusión de la IS entre los Estados miembros (European Union \& The Young Foundation, 2010). Otras entidades en abrir espacio a la IS han sido el Programa de Naciones Unidas para el Desarrollo (PNUD), la Unesco, la Organización de Estados Americanos (OEA), la Secretaría General Iberoamericana (SEGIB), la Comisión Económica para América Latina y el Caribe (CEPAL), y el Banco Interamericano de Desarrollo (BID). También se puede destacar la creación de la Office of Social Innovation and Civic Participation por parte del gobierno de Barack Obama.

Pese a la influencia europea y norteamericana, en Latinoamérica el proceso de apropiación y redefinición del concepto de IS ha tenido ciertas características propias. A continuación resaltamos algunos factores a tener en cuenta de cara al análisis que se espera para la región.

\footnotetext{
${ }^{2}$ La Comisión creó la Oficina de Asesores de Política Europea (BEPA, por sus siglas en inglés), que tiene como fin mejorar la innovación social en las políticas y programas de la Unión Europea.
} 


\section{La Innovación social en Latinoamérica: ¿nuevas respuestas a viejos desafíos?}

Durante las décadas de 1960 y 1970, con programas como la Alianza para el Progreso, se afianzó en Latinoamérica un modo de cooperación internacional asistencialista (García, 2017), que hacía juego a las también asistencialistas políticas sociales de los estados latinoamericanos, y a la perspectiva de asistencia social cristiano-católica que por entonces penetraba ampliamente en las zonas rurales del subcontinente (Castro, 2014). De acuerdo con Domanski, Howaldt \& Schröder (2017), el asistencialismo se caracteriza por tener esquemas organizados para la entrega de bienes y servicios a una comunidad que opera como receptor pasivo, y no se preocupa por empoderarla y capacitarla para enfrentar sus desafíos a través de soluciones construidas de abajo hacia arriba.

A lo largo de las décadas de 1980 y 1990, los países latinoamericanos experimentaron fuertes transformaciones sociales, económicas y políticas derivadas de la crisis de deuda externa y las políticas impuestas por el Fondo Monetario Internacional y el Banco Mundial a través del condicionamiento al préstamo de ayuda financiera (Gordon, Becerra \& Fressoli, 2017; Ocampo, 2014; Rodríguez, 2020). Este paquete de medidas, bautizado por el economista británico John Williamson como Consenso de Washington, propugnaba por el equilibrio presupuestal, la restricción y focalización del gasto público en subsidios a los más pobres, la liberalización económica con respecto al comercio y la inversión, la supresión de regulaciones en los sistemas financieros, la reducción del Estado y privatización de empresas y servicios públicos, el fortalecimiento de los derechos de propiedad, y, en general, la expansión de las fuerzas del mercado dentro de la economía interna (Williamson, 2004).

Las reformas estructurales asociadas a la implementación de estas políticas afectaron la vida económica y social de la región derivando en un aumento de la pobreza, el desempleo y la desigualdad social, lo cual fue denunciado por organizaciones internacionales de las Naciones Unidas como la UNICEF, el PNUD y la CEPAL (Moreno-Brid, Pérez \& Ruiz, 2004; Rodríguez, 2020). En 1995, durante la primera Cumbre Mundial sobre Desarrollo Social, se llamó la atención sobre el deterioro social, no solo en Latinoamérica, sino en todo el mundo: "el rápido proceso de cambio y ajuste se ha visto acompañado de un aumento de la pobreza, el desempleo y la desintegración social" (Naciones Unidas, 1995, p. 7). La cooperación internacional de los países centrales hacia los latinoamericanos asumió entonces la figura de subvenciones a organizaciones no gubernamentales de desarrollo (ONGD) para que se encargaran de programas sanitarios y educativos que los Estados habían dejado de atender (Rodríguez, 2020).

No obstante, la sociedad civil, las comunidades y algunos gobiernos territoriales también se pusieron manos a la obra, y es en esta época de crisis que surgen en Latinoamérica diversas iniciativas que posteriormente, tras un proceso de evolución, serían consideradas innovaciones sociales. Tal es el caso de Ecoorgánica y los Programas de Transferencias Condicionadas (Brasil), TECHO (Chile), 
Programa Integrado de Cultivos Andinos (Argentina), Fortalecimiento de las Finanzas Populares en Azuay y Cañar (Ecuador), Programa Pro-Huerta (Argentina), y Programa de un Millón de Cisternas (Brasil). Los dos últimos fueron estudiados por Gordon et al. (2017), quienes señalan que en Brasil y Argentina "el período neoliberal de la década de 1990 actuó como un enorme laboratorio de movimientos sociales y estrategias de contestación y de $\mathbb{S}$, que luego encontrarían un nicho de desarrollo durante la década de 2000" (Three case studies from South America, párrafo 1).

A principios del siglo XXI los países miembros de Naciones Unidas reaccionaron estableciendo un acuerdo que se expresó en los Objetivos de Desarrollo del Milenio (ODM), en los que la erradicación de la pobreza encabezaba la lista de preocupaciones. A su vez, se llamó a una mayor participación de la empresa privada en el desarrollo social a través del fortalecimiento de estrategias como la Responsabilidad Social Empresarial (RSE), y a una mayor colaboración intersectorial para el cumplimiento de los ODM, lo que dio lugar a las alianzas público-privadas para el desarrollo (Fundación Codespa, 2013; Rodríguez, 2020). En Latinoamérica se dieron avances en la institucionalización de la IS bajo esta perspectiva, la cual fue adoptada con fuerza por países como Chile, donde las alianzas público-privadas a nivel país venían operando (Agencia Chilena de Cooperación Internacional para el Desarrollo [AGCID Chile], 2013; Organización de las Naciones Unidas para la Alimentación y la Agricultura [FAO], 2013). Esto también dio pie a hitos regionales como el Proyecto de Experiencias en Innovación Social en América Latina y el Caribe (PEIS), llevado a cabo por la CEPAL y la Fundación W.K. Kellogg entre 2002 y 2010 (Rodríguez, 2020). En algunos países de la región en los que la crítica al anterior modelo neoliberal se había fortalecido durante gobiernos de izquierda, las políticas públicas retomaron centralidad y la institucionalización de la IS se abrió paso a través de la adopción, como programas de Estado, de innovaciones sociales surgidas en el período de crisis (Gordon et al., 2017). Todo ello se vio beneficiado por un cierto alivio fiscal debido a la bonanza del precio de las materias primas, en cuya exportación han centrado los países latinoamericanos buena parte de su estrategia de desarrollo económico y bienestar (Ocampo, 2008).

Pese a los avances, en Latinoamérica siguen existiendo necesidades sociales insatisfechas o insuficientemente cubiertas, especialmente en los planos de educación, salud y seguridad social, integración económica, empleo, inclusión social, cobertura de servicios públicos, protección del medio ambiente, e igualdad de género (Arenilla, 2017; Domanski et al., 2017; Parada et al., 2017). Además, tras una nueva vuelta de tuerca derivada de la crisis económica y financiera iniciada en 2008 -que se manifestó en la región bajo la forma del retraimiento de la demanda global y la bajada del precio de las materias primas-, se ha visto de nuevo reducido el gasto público, provocando una contracción de la expansión de los servicios públicos, y el incremento de las brechas sociales y de la pobreza (Arenilla, 2017).

En este nuevo escenario la IS se ha seguido fortaleciendo y proyectos de investigación que se han ocupado de Latinoamérica, como el SI-DRIVE, han detectado una actividad creciente en IS en la 
región, especialmente en países como Brasil, Colombia, Chile y Argentina (Domanski et al., 2017). De acuerdo con estas investigaciones, la IS en la región sigue siendo un área que depende principalmente de la participación de las ONG y las comunidades, de manera que las iniciativas más visibles han surgido de estos actores. Aun así, también cabe mencionar el creciente involucramiento de las universidades, lo cual se puede observar en proyectos internacionales que abarcan la región, como Latin American Social Innovation Network (LASIN) o Students4Change.

No obstante, los gobiernos de diferentes países han fortalecido las bases institucionales de la IS, y en países como Brasil se observan ejemplos de participación exitosa de gobiernos en el nivel local, mientras que en otros, como Colombia, se ha dado un papel destacado a la IS en la estructura y políticas de Estado. En este último caso es de resaltar la creación, en 2011, del Centro de Innovación Social adscrito a la Agencia Nacional para la Superación de la Pobreza Extrema (ANSPE), así como el diseño participativo de la Política Pública de Innovación Social, y el establecimiento del Nodo Nacional de Innovación Social (NNIS) como un espacio de acción conjunta entre el gobierno nacional, la ciudadanía, las comunidades y otros actores (Caicedo \& Frías, 2016).

De esta manera, en Latinoamérica vienen proliferando las llamadas antenas de IS, que son descritas por Buckland \& Murillo (2014) como "organizaciones que dedican una parte de sus recursos financieros, además del conocimiento técnico, el asesoramiento y el networking, a promover la actividad de Innovación Social en América Latina" (p. 43). Como señalan estos autores, el espectro va desde organizaciones consolidadas, como Avina y Ashoka, hasta nuevos actores, como el Sistema B que impulsa la certificación en la región de empresas de doble impacto. Estas antenas apoyan a los emprendedores sociales y las empresas por medio de financiación, investigación o creación de plataformas para la IS, y algunas redes de base ofrecen servicios directos a los emprendedores sociales, que van desde espacios de incubación hasta programas de formación. En este escenario, sin embargo, el papel de la empresa privada aún no está muy claro en la región, y tiende a ser definido a través de actividades de RSE (Domanski et al., 2017).

El proceso descrito marca rasgos definitorios de la IS en Latinoamérica que la diferencian de los enfoques de Europa y Norteamérica, pese a la gran influencia conceptual y metodológica que han tenido los países del norte en la región. Los estudios realizados en el subcontinente muestran, por ejemplo, que las prioridades no son las mismas, y, mientras en la Unión Europea se da especial importancia al empleo, en Latinoamérica, las iniciativas se centran en la lucha contra la pobreza extrema. Otras áreas prioritarias de actuación en la región son la inclusión social, igualdad e integración; la educación; la salud y asistencia sanitaria; y, por supuesto, el empleo (Domanski et al., 2017; Parada et al., 2017).

Por último, la pandemia de Covid 19 nos sitúa ante un nuevo panorama, y se hace necesario estar atentos a las nuevas respuestas que traen los principales actores de la IS en Latinoamérica, y 
también en el resto del mundo. La innovación en campos como la educación, la salud, la alimentación, el cuidado de la infancia, el bienestar y prevención de enfermedades mentales, y el bienestar financiero, entre otros, parecen adquirir relevancia en las nuevas circunstancias. Así mismo, será importante observar tendencias como la desterritorialización de las redes de IS.

\section{Pero... ¿qué es esto de la innovación social?}

Más allá de la importancia que ha cobrado la IS, ¿cómo se entiende este término? ¿cuáles son los lindes de la llamada innovación social? La respuesta a estas preguntas aún se está construyendo, y, por eso, la mayoría de los estudios bibliográficos y teóricos comienzan señalando que, pese a la creciente proliferación de trabajos acerca del tema, se trata de un campo que adolece de claridad conceptual e investigativa, y, por tanto, el uso del término sigue siendo ambiguo (Buckland \& Murillo, 2014; Butzin et al., 2014; Jessop et al., 2013; Özbağ et al., 2019; Pacheco et al., 2018). Así, la IS puede concebirse como un proceso, una solución, una metodología, un producto, o como una estrategia de cambio social, entre otros (Castro-Arroyave \& Duque-Paz, 2020).

Como muestran Hernández-Ascanio et al. (2016), una perspectiva diacrónica permite analizar la inestabilidad del concepto, ya que a lo largo del tiempo se han ido sucediendo una serie de definiciones que se han visto afectadas por el momento histórico en el que emergieron, impregnadas, por tanto, de los valores proyectados por los agentes sociales que las proponían. En términos generales, los autores observan que la noción de IS se centró primero en la aplicación de la innovación técnico-científica a las esferas sociales, luego puso en el centro el cómo las innovaciones responden a problemas sociales, para centrarse posteriormente en los aportes específicos desde el ámbito concreto de la acción social o de las organizaciones sin ánimo de lucro, y, finalmente, en el estudio de la sociedad como contexto de generación de innovaciones sociales.

Atendiendo al análisis diacrónico de Pacheco et al. (2018), la evolución del concepto de IS ha pasado por dos grandes etapas. Inicialmente se encuadró en el ámbito de la sociología política, emergiendo asociado al debate sobre la transformación de la sociedad. En esta primera fase, la IS fue percibida como sinónimo de cambio social y su objeto de análisis fue el Estado o la sociedad en general, lo cual incluyó el estudio del papel de la sociedad civil en los cambios sociales, y la manera en que estos se plantaban contrarios a la dinámica macroeconómica global. Posteriormente, se produciría un descenso en el interés por la dinámica global de la is y el concepto experimentaría una resignificación que conduciría hacia la predominancia del microanálisis. En este nuevo enfoque, en el que se destacan los hoy considerados trabajos fundacionales de Taylor y de Gabor en la década de 1970, el interés se centra en las innovaciones sociales ocurridas en organizaciones específicas o en el contexto de un territorio determinado, sin renunciar necesariamente a la consideración de una "perspectiva transformadora" (Pacheco et al., 2018, p. 316). A partir de entonces se da una apropiación 
del concepto de IS y de su universo de términos asociados por parte de diversas disciplinas, factor que, como se verá, ha contribuido a la expansión exponencial de la investigación sobre sobre el tema desde inicios del siglo XXI.

Pacheco et al. (2018) basan buena parte de esta síntesis en las consideraciones publicadas en el libro The International Handbook On Social Innovation: Collective Action, Social Learning and Transdisciplinary Research, publicado en 2013, en el que participan reconocidos autores del campo como Moulaert y Jessop. Estos investigadores se preocupan por recalcar cómo el término IS ha sido utilizado de muy diversas maneras a lo largo de la historia, incluso para abarcar contenidos o fenómenos que difícilmente podrían clasificarse hoy como innovaciones sociales. Entre tanto, otros autores habrían abordado las dimensiones de la IS sin necesidad de usar el término. Según Mumford (como se citó en Moulaert, MacCallum et al., 2013), esto último permitiría remontar el concepto, al menos, hasta el siglo XVIII, cuando Benjamin Franklin evocaría la IS en las innovaciones que propuso en la organización social de la Filadelfia de entonces. Ya en el siglo XIX e inicios del siglo XX, se acuñarían conceptos que hoy se pueden relacionar con la IS, como los de invención social, empleado por autores como Weber, Ogburn, Coleman y Conger, cambio social, transformación o regulación, utilizados por Weber y Durkheim, difusión social a través de la imitación de nuevas prácticas, introducido por Tarde, o cambios en la práctica social (Jessop, et al., 2013).

No obstante, Jessop, Moulaert y sus colegas plantean que los primeros en utilizar el término específico innovación social habrían sido los miembros del movimiento intelectual francés Temps des Cerises en sus análisis y debates sobre la transformación de la sociedad y, en particular, sobre el papel de las revueltas en la Francia de la década de 1960. Posteriormente, Chambon, David y Devevey (1982, como se citó en Jessop et al., 2017) analizarían la relación entre la IS y las presiones provocadas por los cambios sociales, y mostrarían cómo los mecanismos de crisis y recuperación provocan y aceleran la IS. De acuerdo con los autores, estos debates habrían sido, a la vez, la bisagra entre las "viejas teorías del cambio social" (Jessop et al., 2013, p. 113) y los nuevos enfoques de la IS, que iniciarían en la década de 1970 y se desarrollarían especialmente desde finales de la década de 1980:

Aunque varios analistas contemporáneos continúan trabajando en la "vieja" tradición, la mayoría de ellos ahora están más inclinados a utilizar un enfoque más micro-lógico, parcial u orientado a la práctica. El reciente giro hacia la IS como un vehículo clave en el "liberalismo solidario" que privilegia la empresa social como agente del cambio social y la economía como la esfera principal de la vida social, puede conectarse con este cambio de orientación. (Jessop et al., 2013, p. 113).

De esta manera, desde finales de la década de 1980, la investigación de la IS se habría alejado de manera importante de la dinámica del proceso social como foco de interés, y se habría centrado en la relación fin-medios -por ejemplo, una junta de vecinos que establece acciones para superar la exclusión social-, quedando espacio para los enfoques "macro" sólo en algunos campos que cruzan los límites disciplinarios estándar. Como consecuencia, estos autores distinguen críticamente dos grandes perspectivas actuales de trabajo en la IS: por un lado, sigue siendo una idea de referencia para los 
movimientos sociales y políticos que persiguen el desarrollo humano, y, por otro, la IS es cada vez más adoptada para resolver la crisis del estado de bienestar: un "política rápida" de "soluciones milagrosas" que busca la creación de nuevos puestos de trabajo en la economía social "barata" y "reorganizando el sistema de bienestar a través de la mercantilización y privatización de algunos de sus servicios y la reestructuración más eficiente de otros" (Moulaert, MacCallum et al., 2013, pp. 17-18).

Son varios los autores que, aunque con un acento menos crítico, observan una polaridad similar en el actual enfoque de investigación y teorización de la IS. Martínez et al. (2019), por ejemplo, reúnen las formas de aproximación en dos grandes ejes: uno de corte "economicista" (p. 4), que considera que la IS reside principalmente en el individuo emprendedor y busca llenar vacíos dejados por el Estado en cuanto a la resolución de demandas sociales. Así, las innovaciones sociales, para ser consideradas como tales, no tendrían que generar un cambio en las relaciones de poder o transformaciones institucionales, "sino que lo esencial radica en su capacidad para resolver problemas concretos a través de nuevos productos o servicios" (p. 4). El otro enfoque es el que Oosterlynck et al. (como se citó en Martínez et al., 2019, p. 5) llaman "el enfoque de base de la innovación social", en el que esta última resulta de la movilización de fuerzas sociales e institucionales que buscan satisfacer necesidades humanas hasta ahora alienadas, e implica el empoderamiento de grupos excluidos 0 silenciados a través de la creación de nuevas capacidades, todo lo cual lleva a la transformación de las relaciones sociales hacia sistemas de gobernanza más democráticos e inclusivos.

Van der Have \& Rubalcaba (2016), por su parte, hablan de una aproximación sociológica que contrasta con una más económica. La primera se centra en las prácticas sociales y concibe la IS como nuevas formas de crear e implementar el cambio social. Los autores identificas algunos teóricos preeminentes en este enfoque, como Moulaert y sus colegas de la escuela francesa, quienes distinguen tres dimensiones que interactúan en la IS, como son la satisfacción de las necesidades humanas, los cambios en las relaciones sociales, y el empoderamiento de los actores sociales. También destacan a Hochgerner, y Howaldt \& Schwarz, quienes ven la IS como una nueva combinación de prácticas sociales, y la entienden como un nuevo paradigma de innovación, en lugar de una categoría separada de la misma. Para Van der Have \& Rubalcaba, las conceptualizaciones económicas, en cambio, están más orientadas a los resultados y se relacionan con las "ideas", los "servicios" o las nuevas transformaciones "sistémicas" y los impactos sociales asociados. Aquí destacan los trabajos Pol \& Ville, y también inscriben las organizaciones supranacionales que definen la IS como nuevas soluciones de servicios, cual es el caso de la OCDE.

Al interior, en la intersección y entre medio de estos grandes enfoques polarizados de aproximación a la IS, emergen numerosas definiciones. El estudio realizado por Hernández-Ascanio et al. (2016), por ejemplo, se centró en 62 artículos de WoS y Scopus que contenían una definición explícita de IS, localizando 48 conceptos diferentes. Según los autores, esta vaguedad y confusión estaría relacionada con la variedad de disciplinas o campos que han apropiado el término, entre las 
que se pueden destacar las ciencias de la administración, gestión y economía, emprendimiento social, sociología, psicología, antropología social, ciencias políticas, tecnología, diseño, artes y creatividad, y ecología, entre otras (Özbağ et. al., 2019; Pacheco et al., 2018; Weerakoon et al., 2016).

A continuación, se darán más detalles de los enfoques de investigación y se brindará un panorama acerca de la publicación científica.

\section{Panorama de la investigación y la publicación científica en innovación social}

No obstante que, como se ha dicho, algunos estudiosos de la IS consideran que existen antecedentes relevantes para la construcción teórica del campo actual en las obras de autores como Weber o Durkheim, es importante señalar que el primer estudio que aparece en revistas científicas empleando el término innovación social es el publicado por Garvey and Griffith (1966), titulado Studies of social innovations in scientific communication in psychology. Aun así, los trabajos más relevantes que se relacionan con el actual concepto de IS son Introducing Social Innovation (Taylor, 1970), y Innovations: scientific, technological and social (Gabor, 1970). A partir de estas publicaciones la IS gana importancia como tema de estudio, y desde 2003 hay un crecimiento exponencial de la publicación de artículos en la materia (ver Gráfico 1).

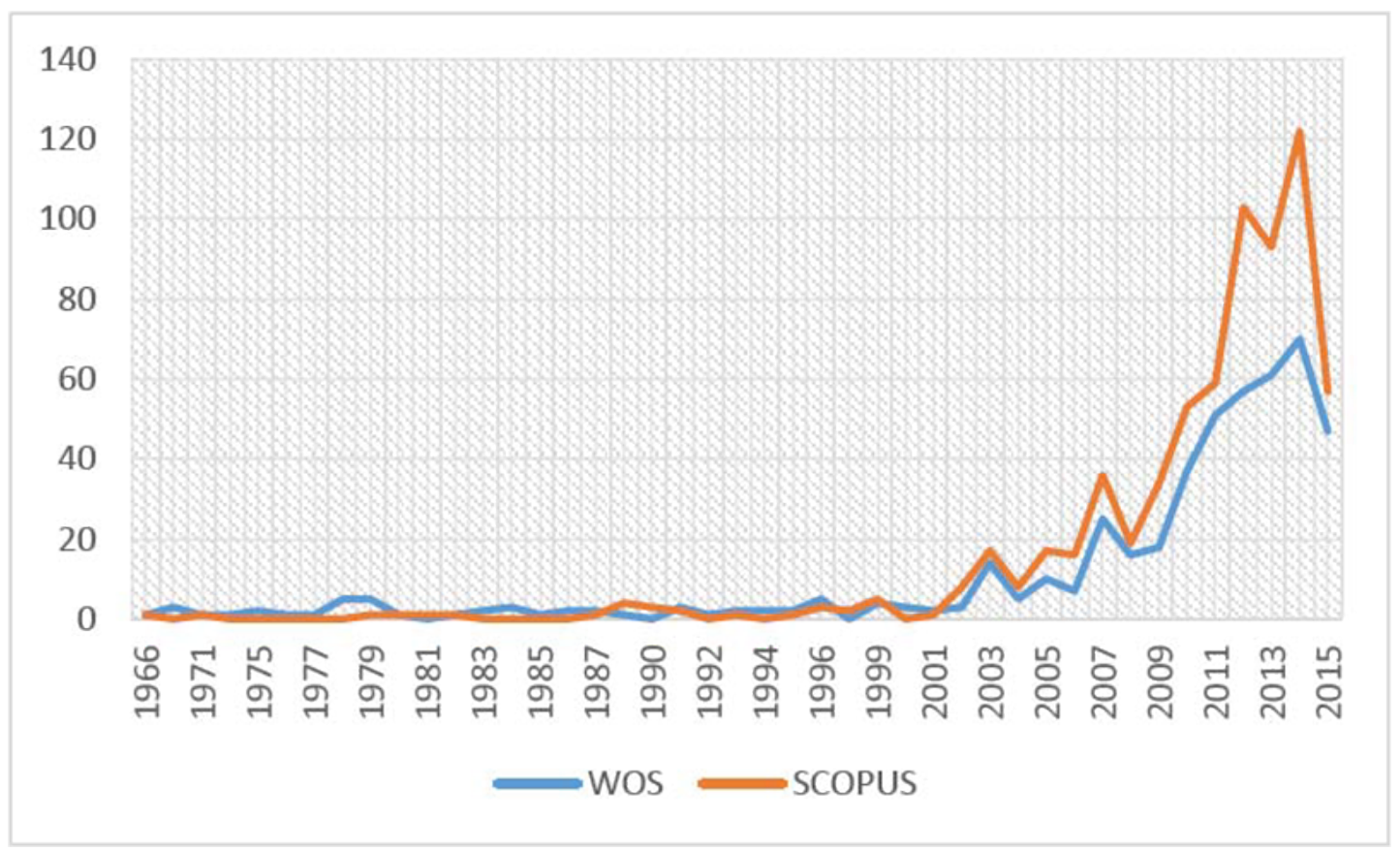

Gráfico 1: Evolución de la publicación sobre IS en revistas indexadas en WoS y Scopus (1966 - 2015) (Fuente: Pacheco et al., 2016, p. 306). 
Como se ha dicho, este crecimiento del interés académico-científico en la IS está relacionado con la apropiación del tema por campos de disciplinas diversas. Quienes han realizado estudios teóricos y bibliográficos han encontrado algunos conjuntos de áreas temáticas que clasifican con algunas variaciones. La clasificación realizada por Pacheco et al. (2018), que integra propuestas precedentes de Moulaert, Jessop y sus colegas, reconoce cinco líneas fundamentales de investigación: 1) Ciencias de la gestión y la economía; 2) Artes y creatividad; 3) Ciencias políticas, gestión pública y participativa, que también incorpora el campo del desarrollo local; 4) Estudios de emprendimiento social; 5) Una línea discontinua de investigación en psicología.

Van der Have \& Rubalcaba (2016), por su parte, sugieren que el campo de la IS se basa en cuatro comunidades intelectuales distintas que surgen a través de un proceso de difusión algo organizado: 1) Psicología comunitaria; 2) Investigación de la creatividad; 3) Desafíos sociales; 4) Desarrollo local. De acuerdo con los autores, el interés por la IS en las áreas de gestión y emprendimiento es muy reciente y actualmente se refleja en comunidades inexistentes.

Westley (2013), por su parte, más que disciplinas o líneas de investigación, ha identificado seis escuelas de pensamiento distintivas que incluyen: 1) Emprendimiento social; 2) Teoría de la innovación; 3) Emprendimiento institucional; 4) Transiciones socio-técnicas; 5) Interacciones de escalas múltiples y cruzadas; 6) Resiliencia socio-ecológica; 6) Economía social. Phillips et al. (2015, como se citó en Özbağ et al., 2019), en cambio, identifican tres principales campos de investigación: 1) Papel del emprendedor; 2) Relaciones de asociación; 3) importancia del entorno institucional.

A nivel internacional, en todos estos campos o disciplinas existe un protagonismo de autores europeos y norteamericanos. Tanto el incremento como esta distribución geográfica de las publicaciones en materia de IS es resultado de la influencia de las agendas de políticas de organismos internacionales y gobiernos en los Estados Unidos y la Unión Europea, especialmente tras la crisis económica y financiera iniciada en 2008 (Pacheco et. al, 2018). En el caso de Europa, se ha dado una importante financiación para la creación y fortalecimiento de institutos, y para el desarrollo de proyectos de investigación, especialmente tras el aumento de su promoción en el Séptimo Programa Marco de Investigación y Desarrollo Tecnológico (7PM), el cual lanzó varias convocatorias entre 2007 y 2012 (Domanski \& Kaletka, 2016). Por su parte, la alta participación de académicos estadounidenses a partir de 2010 también estaría relacionada con la inclusión de la IS en las agendas de política social y la creación de instancias específicas para su gestión en la estructura gubernamental, como respuesta a la recesión económica después de 2008 (Weerakoon et al., 2016).

Este impulso llevó al desarrollo de proyectos de investigación de alcance mundial, como Social Innovation: Driving Force for Social Change (SI-DRIVE) y Transformative Social Innovation Theory (TRANSIT), ambos financiados por la Comisión Europea. El primero buscaba contribuir a una mejor comprensión de la IS describiendo a escala mundial el fenómeno en toda su diversidad "para desarrollar modelos robustos para la creación, implementación y difusión de innovaciones sociales", y 
"para comprender mejor la relación entre innovación social y cambio social" (Howaldt, Domanski \& Kaletka, 2016, p. 30). El segundo fue un ambicioso proyecto que se basó en la retroalimentación de emprendedores e innovadores sociales, responsables políticos, y académicos, para desarrollar una teoría sobre la IS de relevancia práctica y carácter transformador, es decir, enfocada en el empoderamiento y el cambio en la sociedad (http://www.transitsocialinnovation.eu/).

Estos proyectos han arrojado luces sobre otras regiones del mundo donde la investigación en IS está bastante menos desarrollada, como es el caso de Latinoamérica. Como señalan Domanski et al. (2015), quienes participaron en el SI-DRIVE, la investigación en IS en la región se encuentra en una etapa inicial y existe aun relativamente poca literatura sobre el tema, de manera que "se requiere un análisis sostenido y sistemático de la innovación social y su relación con el cambio social transformador" (p. 49). Como se verá en el siguiente apartado, los países que más destacan en la dimensión investigativa de la IS en la región son, en primera instancia, Brasil y Colombia, seguidos de lejos por México. Aun así, las publicaciones de autores y revistas latinoamericanos también se han visto sensiblemente incrementadas desde 2003, y esto, según Van der Have \& Rubalcaba (2016), tiene que ver con factores que exceden lo meramente político, y que operan a nivel general, como el compromiso de los ciudadanos y las organizaciones con la innovación, la crítica a los modelos comerciales dominantes, las grandes caídas del gasto público, y las necesidades de las economías en desarrollo, donde la innovación no se trata de tecnología de punta, sino de resolver problemas sociales (Van der Have \& Rubalcaba, 2016).

\section{El mapa de las publicaciones científicas}

La preeminencia de Europa y Norteamérica en la dimensión investigativa de la IS se puede observar en el mapa de publicaciones en revistas científicas que abordan la temática. Uno de los pocos estudios que integran las dos bases de datos más grandes, WoS y Scopus, es el realizado por Pacheco et al. (2018), en el cual se puede observar la predominancia de los países anglosajones, sobre todo Estados Unidos, Reino Unido y Canadá. En consecuencia, las universidades más destacadas en producción investigativa en la materia están, en general, en estos países, siendo las primeras la de Oklahoma, Waterloo, Quebec, Newcastle y, como excepción, Milán, en el caso de la WoS, y las universidades de Quebec, Waterloo y Newcastle en Scopus. En consecuencia, el idioma inglés también resulta predominante y constituye el $80 \%$ de los trabajos en el WoS y el $86 \%$ en el caso de Scopus.

Un estudio de la WoS más reciente y detallado en aspectos bibliométricos es el realizado por Özbağ et al. (2019) que concluye que los países que más publicaciones tienen en la materia son Reino Unido, Estados Unidos e Italia. Para el caso de Scopus también resulta más minucioso en aspectos bibliométricos el estudio realizado por Weerakoon et al. (2016), que reafirma que la distribución de publicaciones entre países está muy desequilibrada, ya que Estados Unidos, Reino Unido y Canadá representan casi el 76\% de las 949 publicaciones halladas por los autores, y 24\% restante se distribuye entre 72 países. 
Los países latinoamericanos, en general, no destacan en la publicación científica internacional acerca de la IS, a excepción de Brasil, que aparece ocupando el décimo puesto en el estudio correspondiente a la WoS. Es necesario hacer foco en bases de datos y directorios especializados en publicaciones iberoamericanas para encontrar la producción científica regional en la materia. Con este fin, y sin que se trate de un estudio exhaustivo, se realizó una exploración en el SciELO Citation Index utilizando como criterio de búsqueda el término "innovación social" en el tema (título, resumen y palabras clave). Se recuperaron 108 publicaciones, el 100\% de las cuales son artículos científicos. ${ }^{3}$ Las publicaciones sobre IS en SciELO inician en 2003, y comienzan a tener mayor presencia desde 2012 (Ver Gráfico 2).

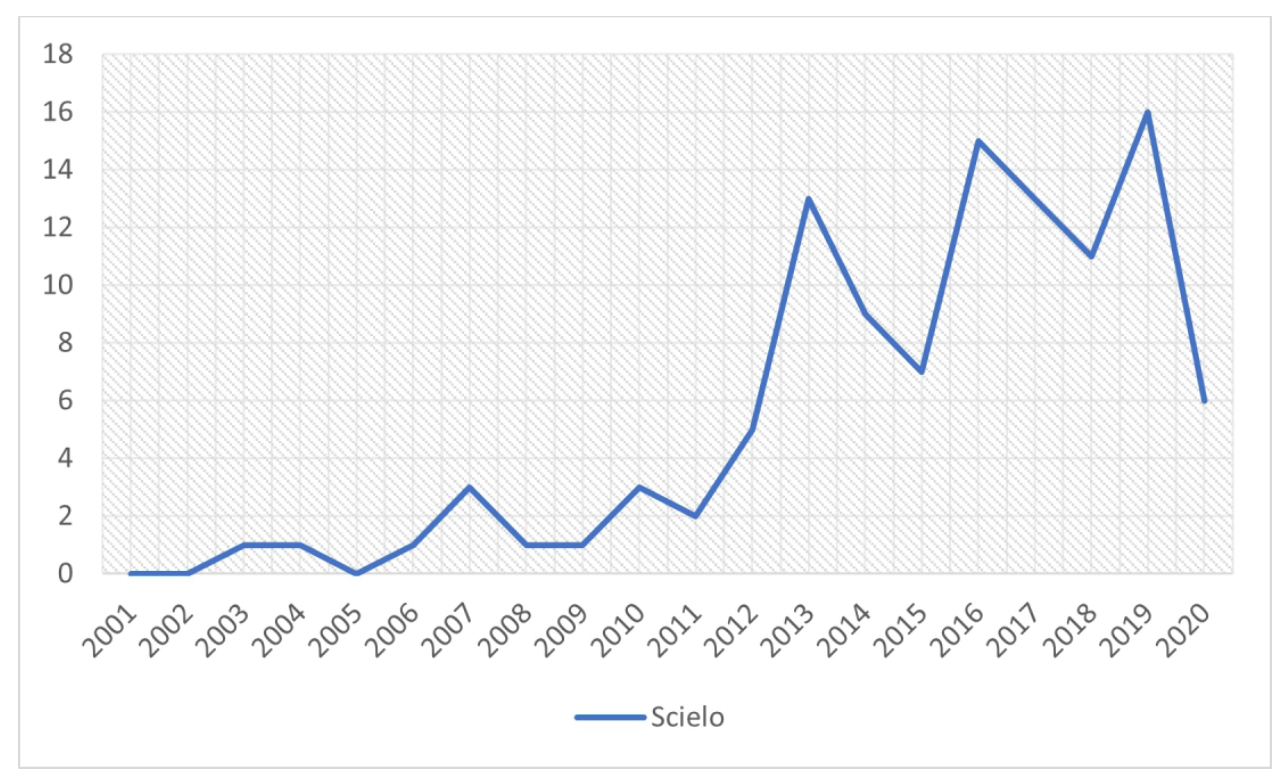

Gráfico 2: Evolución de las publicaciones sobre IS en SciELO. (Elaboración propia).

En cuanto a distribución geográfica, los países con el mayor número de publicaciones son Brasil (25,9\%), Colombia (20,3\%), México (11,11\%) y Portugal (10,1\%), cada uno con 28, 22, 12 y 11 publicaciones, respectivamente. El 50,9\% de estos trabajos se publicó en español, el 28,9\% en portugués, el 22,2\% en inglés, y el 0,92\% en francés. Si se hace foco en las instituciones de afiliación, se observa que las universidades que más destacan por el número de publicaciones son la Universidad de Lisboa (Portugal), Universidade do Estado de Santa Catarina (Brasil), Universidad de Antioquia (Colombia), Universidad Nacional Autónoma de México, Universidad Nacional de Colombia, Universidade do Vale do Rio dos Sinos (Brasil) y la Universidade Federal de Minas Gerais (Brasil).

Además, si se observa la distribución por áreas de investigación según categorías del índice, cabe destacar que en Iberoamérica la publicación científica sobre IS se concentra de manera más

\footnotetext{
${ }^{3}$ La exploración tuvo lugar a mediados de 2020, para el inicio del proceso editorial de la revista SIS. Lo anterior explicaría el descenso en el número de publicaciones en 2020 que se observa en la Gráfico 2, ya que no se abarcó el año completo.
} 
marcada que a nivel global en el área de economía y negocios (business economics), con un 37,96\% de los artículos. Las demás áreas de publicación son sociología (sociology, 19,4\%), otras áreas de las ciencias sociales (social sciense other topics, 11,1\%), ingeniería (engineering, 7,4\%), artes y humanidades (art humanities other topics, 6,48\%), legislación y gobierno (government law, 6,48\%), administración pública (public administration, 5,5\%), geografía (geography, 4,6\%), asuntos sociales (social Issues, 3,7\%) y estudios urbanos (urban studies, 3,7\%), entre las áreas más destacadas.

Una conclusión relevante de los estudios bibliométricos realizados sobre bases de datos como Wos, Scopus y SciELO, es que las publicaciones científicas sobre IS se encuentran diseminadas en un gran número de revistas de diversas disciplinas, no obstante que destacan algunas en cuanto a la concentración de artículos sobre el tema. La explicación que dan los autores de estos análisis es que no existen revistas científicas especializadas en IS que estén indexadas en estas bases de datos (Özbağ et. al., 2019; Weerakoon et al., 2016). ${ }^{4}$

En el estudio de WoS (1975-2018) de Özbağ et. al. (2019), los autores muestras que, de 1.196 publicaciones halladas según sus criterios de búsqueda, 725 se encuentran en diferentes fuentes. Para el caso de este estudio las tres revistas con el mayor número de publicaciones pertenecen a tres disciplinas distintas: Design Journal (40), Innovation - The European Journal of Social Science Research (17) y PICMET (Portland International Conference on Management of engineering and technology) 2016 (14).

En el estudio de Scopus, realizado por Weerakoon et al. (2016), también se observa una diversidad de disciplinas entre las revistas con mayor número de publicaciones en la materia, que fueron Hitachi Review (24), Journal of Social Entrepreneurship (16), y Futures (10) y Salute E Societa Health and Society (10), que empatan en la tercera posición.

Para el caso de SciELO, entre las revistas con mayor número de publicaciones en la materia se pueden destacar la RAM Revista de Administracao Mackenzie (U. Presbiteriana Mackenzie, Brasil), la Revista Iberoamericana de Ciencia, Tecnología y Sociedad (Conicet, Argentina), la Journal of Technology Management \& Innovation (U. Alberto hurtado, Chile) y la Finisterra Revista Portuguesa de Geografía (U. de Lisboa, Portugal), observándose también una multidisciplinariedad en la publicación iberoamericana en IS.

Esta atomización también se puede relacionar con que, como señalan Van der Have y Rubalcaba (2016), el área de estudio de la SI aún no está bien integrada y consolidada como campo de investigación, lo cual complica la acumulación sistemática de conocimiento y el crecimiento de un

\footnotetext{
${ }^{4}$ Al momento de la realización de estos estudios, la European Public \& Social Innovation Review (EPSIR), publicada por Sinnergiak Social Innovation (Universidad del País Vasco), se encontraba apenas en proceso de indexación en Scopus, por lo que no figura en los hallazgos. Esta es la única revista científica existente dedicada explícitamente a la innovación social. Se trata de una revista bianual, interdisciplinaria, con revisión de pares y de acceso abierto, que se publica desde 2016 y en 2021 ha llegado a su sexto volumen.
} 
campo de investigación emergente. De acuerdo con los autores citados, esto plantea un desafío para que los académicos desarrollen conocimientos generalizables y formulen teorías e hipótesis articuladas sobre la IS. Así, se entiende la predominancia del análisis de casos de estudio en la investigación de la IS, lo cual refleja, a su vez, lo señalado por Jessop et al. (2013) acerca del paso de un enfoque macro a uno micro en el estudio de la IS.

\section{Perspectivas de Social Innovation Studies}

La vaguedad conceptual que hasta el momento ha sido característica del campo de la IS ha llevado a algunos académicos a cuestionar el origen y definición de este fenómeno (Consiglio \& Zabatino, 2015). SIS considera que el término, pese a su actual indefinición, tiene la capacidad de apuntar a procesos sociales que se vienen solidificando en un contexto internacional de mayor participación de la sociedad en la búsqueda de soluciones para sus propias problemáticas, y que, de alguna manera, agrupa fenómenos sociales, culturales, económicos y políticos contemporáneos que es importante atender y descifrar. Así, estamos de acuerdo con Moulaert et al. (2013) en que:

En todos los continentes, la mayoría de los países, regiones, ciudades o incluso distritos urbanos donde han surgido problemas u oportunidades de mejora socio-económico-político-culturalambiental que tocan las condiciones existenciales y de vida de las personas, han surgido enfoques y soluciones socialmente innovadores que comparten un perfil común, en el que el empoderamiento social y político de las personas es fundamental para satisfacer sus necesidades insatisfechas; en el que la mejora de las condiciones materiales y el cambio de las relaciones sociales están íntima y necesariamente conectados. En consecuencia, la innovación social también puede verse como una "conciencia" general y compartida sobre la naturaleza de los problemas a los que se enfrentan las sociedades modernas y las formas en que deben afrontarlos. (pp. 2-3).

De esta manera, y siguiendo el planteamiento de Castro-Arroyave \& Duque-Paz (2020), consideramos que la falta de especificidad conceptual, sumada al interés por el tema, antes que negar la existencia de la IS sugieren que se trata de un concepto en construcción, y SIS quiere asumir el desafío de abordar este proceso en toda su complejidad. Lo anterior, teniendo en cuenta que, como han señalado varios estudios (Özbağ et al., 2019; Weerakoon et al., 2016), la falta de revistas especializadas y la debilidad de redes de coautoría son factores que pueden haber incidido en la ambigüedad conceptual existente en este campo.

Ahora bien, algunos autores consideran que, debido al enfoque práctico y aplicado se la IS, a su profunda vinculación con el contexto en el que emerge el fenómeno, a la diversidad de desafíos que aborda, a sus numerosos puntos de contacto con terminologías y modelos que habían estado integrados en grandes disciplinas -como el emprendimiento social-, la IS "rehúye todo intento de encapsulamiento en un espacio teórico cerrado y preciso" (Buckland \& Murillo, 2014, p. 6), y tampoco es necesario que exista una única definición de IS, ya que esta se adapta al contexto donde intenta aplicarse (Monge \& Allamand, 2016). 
Teniendo en cuenta estas ideas, el objetivo de SIS no es asumir un enfoque único de la IS ni llegar a asentarse en un concepto monolítico, sino establecer vasos comunicantes entre los distintos enfoques y niveles de abordaje de sus analistas. El objetivo es, entonces, situarse en el corazón del debate en Latinoamérica, invitando a todos los interesados en él a participar a través de este espacio como medio de divulgación científica, pero también, como foro abierto a la reflexión y el diálogo, en el que se encuentren investigadores, académicos, técnicos, gestores de política pública e innovadores sociales. De esta manera SIS se convertirá en un medio de referencia para comprender cómo se entiende y fomenta la IS en el subcontinente, aportando así a la tarea de construir un campo de conocimiento a nivel global.

Sobre esto último, SIS asume el desafío claro de fomentar la investigación y publicación en IS en Latinoamérica. Precisamente porque este es un campo que se ha venido construyendo especialmente desde la práctica, y, de forma concomitante, los gobiernos y organismos multilaterales han tenido gran influencia en la definición de la IS sin que su agenda política esté del todo sobre la mesa, es importante que la academia redoble sus esfuerzos por participar en esta construcción teórica, ya que la IS, como señalan Moulaert et al. (2013), también es un constructo científico que se deslinda a partir del análisis de la esencia de las iniciativas, experiencias y procesos innovadores.

Para lo anterior, lo mismos autores ayudan a distinguir dos importantes características de la investigación en IS que son, a su vez, puntos fuertes que se quieren promover desde SIS: primero, que se trata de un campo idóneo para trabajar de manera trans e interdisciplinaria con posturas epistemológicas y metodológicas que están en continuo desarrollo, y, de esta manera, puede decirse que permiten la innovación en los procesos de investigación; segundo, que la IS lleva a no agotarse en lo teórico ni quedarse en lo puramente científico, pues trata sobre las aspiraciones de desarrollo humano de una diversidad de actores sociales, de manera que tiene una fuerte orientación hacia la acción (Moulaert, MacCallum et al., 2013, págs. 13-14).

Ahora bien, con el propósito de ser, efectivamente, un espacio de encuentro para el atomizado grupo de interesados en la IS en Latinoamérica, SIS está abierta a un amplio espectro de enfoques de estudio, desde los que aquí hemos denominado micro, cuya metodología de investigación se basa especialmente en estudio de casos, hasta las aportaciones con enfoques macro, que buscan regresar a la reflexión sobre la IS en relación con los grandes procesos de cambio social.

Así, creemos que es importante seguir ahondando en la sistematización y análisis, tanto de resultados e impactos, como de procesos de IS. En cuanto a los primeros, han sido los más estudiados, pero sigue siendo relevante atender a los productos, servicios y modelos que derivan de las iniciativas de IS, así como a las capacidades que activan y a las interacciones beneficiosas que generan, ya que todo ello da cuenta, en último término, de la efectividad de la IS y de su importancia como mecanismo de cambio (Bernaola, 2016). Pero específicamente, como enfatizan Buckland \& Murillo (2014), existe un 
interés compartido entre las entidades que apoyan la IS y, en general, entre analistas financieros, políticos, fundaciones, académicos y los propios innovadores sociales, por medir el impacto social de las iniciativas.

La dimensión del proceso, por su parte, cobra gran relevancia para el análisis de la IS, pues, como señalan Rodríguez \& Alvarado (2008), debido a su carácter impetuoso, disruptivo y, en algunas ocasiones, hasta transgresivo, se pasa por alto con frecuencia que cada innovación es el resultado "de un cúmulo de intentos fallidos y pequeñas mejoras que en un momento crítico cambian el signo de la tendencia, la dirección de un proceso, la calidad de un producto o la técnica de un procedimiento" (p. 23). De esta manera, y según los mismos autores, "la originalidad de la innovación radica en el proceso que permite hacer realidad un cambio específico" (p. 23), por lo que es dicho proceso lo que más interesa analizar para comprender el cambio, y, si es que se quiere, observar la posibilidad de replicar o escalar la iniciativa. Buckland \& Murillo (2014) resaltan cómo aspectos del proceso, tales como el modelo de negocio, el tipo de innovación -incremental o radical- o la colaboración intersectorial, resultan de especial interés para quienes están interesados en detectar qué iniciativas de IS tienen la capacidad de escalar y "provocar el cambio sistémico deseado" (p. 12).

Así pues, tanto el análisis de resultados e impactos, como el de procesos, se siguen considerando indispensables y se entienden como necesarios cuando se trata de proyectar las innovaciones sociales al plano de las políticas públicas (Domanski \& Kaletka, 2016; Rey De Marulanda \& Tancredi, 2010). Sin embargo, Gordon et al. (2017) llaman la atención sobre la necesidad de no detenerse allí, sino de hacer seguimiento y analizar críticamente la evolución de las innovaciones sociales como políticas públicas, abriendo paso a la gobernanza e institucionalización de la IS como importante subcampo de investigación.

Ahora bien, en cuanto al estudio empírico de la IS, existen dos focos de interés para SIS, que no deben entenderse como separados, sino que pueden traslaparse: el enfoque de base de la IS (Oosterlynck et al., como se citó en Martínez et al., 2019, p. 5), es decir, la IS que resulta de la movilización de fuerzas sociales e institucionales que buscan satisfacer necesidades humanas hasta ahora alienadas, e implica el empoderamiento de grupos excluidos o silenciados a través de la creación de nuevas capacidades; y aquella IS que surge en procesos multihélice de innovación, en los que gobiernos y sector público, empresa privada, sociedad civil y universidad interactúan, con un rol primordial de la ciudadanía en la que agentes impulsores (organizaciones comunitarias, emprendedores sociales, etc.) emergen para proponer soluciones con impacto social y/o ambiental frente a desafíos detectados en el entorno (Concha \& Ricci, 2018).

Ahora bien, como señalan Rodríguez \& Alvarado (2008), la innovación es, por una parte, un proceso endógeno de autoorganización, pero, por otro, también es "un proceso exógeno, ecoorganizado, que supone factores externos que condicionan el ritmo de la innovación" (p. 24). Esto 
nos lleva a la importancia de atender el contexto, y, así, considerar los planteamientos de Westley \& Antadze (2010) sobre la IS como un sistema y proceso complejo, en el que la acción y el impacto no se rigen por relaciones directas de causa y efecto:

Lograr procesos complejos se parece más a criar hijos: el éxito con uno no es garantía de éxito con otro, y las recetas o los planos tienen un valor limitado. La gestión de una relación emergente y en constante evolución entre padres, hijos y el contexto social más amplio se encuentra en el corazón de este proceso. Los choques o discontinuidades imprevistas pueden descarrilar la relación, cambiando las reglas en cualquier momento. Los resultados siguen siendo inciertos. ( $p$. 13).

En este proceso complejo la agencia juega un rol fundamental para aprovechar las oportunidades, y, en este sentido, la investigación se ha centrado en el emprendedor social: ese agente que tiene la capacidad de trabajar en condiciones de alta complejidad, y, desde allí, iniciar o crear programas, productos o procesos innovadores, buscando construir una organización inicial que pueda llevar esa innovación al mercado. No obstante, Westley \& Antadze (2010) nos recuerdan la importancia de analizar también el papel de los emprendedores institucionales: aquellos individuos o redes de individuos que buscan activamente cambiar el sistema social más amplio a través del cambio de las instituciones políticas, económicas, legales o culturales, a fin de que la IS pueda florecer.

No obstante, como indican estos autores, en sistemas complejos, la agencia no puede explicar ningún cambio por sí sola, sino que debe confluir con la oportunidad, que es una característica del contexto social e institucional más amplio. La IS puede verse favorecida por una demanda del mercado, o una demanda ciudadana, política o cultural, entre otras, por lo que su estudio también implica el análisis acucioso y crítico de los procesos históricos que originan las necesidades sociales que, a su vez, dan pie a las innovaciones sociales (Rodríguez, 2020).

Desde allí, es esperable que, como piden Moulaert y sus colegas, el estudio de la IS en Latinoamérica también se amplíe a enfoques macrosociales, con un compromiso ético por aportar al mejoramiento de las condiciones de vida de los más excluidos:

Si la innovación social se trata de abordar problemas, mejorar la condición humana, satisfacer las necesidades de los humanos, establecer agendas para un futuro mejor, etc., entonces, como concepto científico, debe incluir la búsqueda de la mejora o realización de la existencia humana, un mejor equilibrio en la convivencia, junto con la evolución de las relaciones entre los seres humanos y el inicio de acciones para mejorar la condición humana. (Moulaert, MacCallum et al., 2013, p. 17).

Corroborando lo dicho acerca del contexto, estos autores también señalan que la IS no se puede separar de su contexto sociocultural, o de su contexto sociopolítico, sino que se hace relevante "analizar la relación entre el sistema al que están reaccionando muchos de los actores de la is y el significado político de sus acciones" (Moulaert, MacCallum et al., 2013, p. 18). A su vez, implica un compromiso por desarrollar la investigación en IS de una manera democrática, involucrando a todos los actores interesados en mejorar la condición humana y construyendo modelos de investigaciónacción transdisciplinarios que lo permitan. 
Queda, entonces, abierto el espacio de Social Innovation Studies para todos los que quieran responder a esta invitación y acudir a él con un ánimo colaborativo, con desprendimiento respecto a los protagonismos académicos, científicos, políticos, o de cualquier tipo, y el foco puesto en los desafíos sociales que hoy en día debe abordar la región y que solo será posible asumir a través de la muchas veces aludida, y, por tanto, casi vaciada de sentido, lógica de la co-construcción.

\section{Reconocimientos}

Los autores declaran que no existen conflictos de interés en su texto.

\section{Referencias}

AGCID. Agencia Chilena de Cooperación Internacional para el Desarrollo. (2013, julio 30). Chile promueve modelo de Alianza Público-Privada para la Cooperación al Desarrollo. Ministerio de Relaciones Exteriores. https://bit.ly/3JaKdt6

Arenilla, M. (2017). Innovación social y capacidad institucional en Latinoamérica. Revista del CLAD Reforma y Democracia, 67, 33-68. https://bit.ly/3J69Nzf

Bernaola, G. (2016). Panorama actual de la Innovación Social en Latinoamérica. En D. Domanski, N. Monge, G. Quitiaquez, \& Rocha, D. (Eds.), Innovación Social en Latinoamérica (pp. 21-26). Corporación Universitaria Minuto de Dios, Parque Científico de Innovación Social.

Buckland, H. \& Murillo, D. (2014). La Innovación Social en América Latina. Marco conceptual y agentes. Instituto de Innovación Social de ESADE y Fondo Multilateral de Inversiones - Banco Interamericano de Desarrollo.

Butzin, A., Davis, A.S., Domanski, D., Dhondt, S., Howaldt, J., Kaletka, C., Kesselring, A., Kopp, R., Millard, J., Oeij, P.R., Rehfeld, D., Schaper-Rinkel, P., Schwartz, M., Scoppetta, A., Wagner-Luptacik, P., \& Weber, M. (2014). Theoretical approaches to social innovation - A critical literature review. https://bit.ly/3J8R1Hp

Caicedo, A. \& Frías, N. (2016). Política Nacional de Innovación Social colombiana: el proceso de construcción participativa. En D. Domanski, N. Monge, G. Quitiaquez, \& Rocha, D. (Eds.), Innovación Social en Latinoamérica (pp. 193-206). Corporación Universitaria Minuto de Dios, Parque Científico de Innovación Social.

Castro, B. (2014). La relación entra la iglesia católica y el Estado Colombiano en la asistencia social: 18791960. Editorial Universidad del Valle.

Castro-Arroyave, D.M. \& Duque-Paz, L.F. (2020). Documentary research on social innovation in health in Latin America. Infect Dis Poverty, 9(41). https://doi.org/10.1186/s40249-020-00659-6

Comisión Europea. (2014). Horizon 2020 en breve. El Programa Marco de Investigación e Innovación de la UE. El autor. https://bit.ly/3yPmHx6 
Concha, R. \& Ricci, E. (2018). Innovación Social Multihélice en la Región de Antofagasta. En E. Ricci, E. \& R. Concha (Eds.), Innovación Social. Consolidación Modelo Multihelice en la Región de Antofagasta (pp. 23-41). Ediciones Universidad Católica del Norte.

Consiglio, S. \& Zabatino, S. (2015). L'innovazione sociale per la rinascita del patrimonio dimenticato. En S. Consiglio S. \& A. Riitano (Eds.), Sud Innovation. Patrimonio Culturale, Innovazione Sociale e Nuova Cittadinanza (pp. 69-102). Franco Angeli.

Domanski,D., Howaldt, J., \& Schröder, A. (2017). Social Innovation in Latin America. Journal of Human Development and Capabilities, 18(2), 307-312. https://doi.org/10.1080/19452829.2017.1299698

Domanski, D., Howaldt, J., Villalobos, P. \& Huenchuleo, C. (2015). Social Innovation in Latin America: The Chilean Case. Cieplan. https://bit.ly/3FipgKp

Domanski, D. \& Kaletka, C. (2016). La investigación en innovación social en la Unión Europea y la experiencia de la TU Dortmund University- sfs. En D. Domanski, N. Monge, G. Quitiaquez, \& D. Rocha (Eds.), Innovación Social en Latinoamérica (pp. 209-232). Corporación Universitaria Minuto de Dios, Parque Científico de Innovación Social.

European Union \& The Young Foundation. (2010). Study on Social Innovation. Los autores.

García, M.A. (2017). Modernizar la tradición. Carlos Rojas en Artesanías de Colombia durante los años 70. Nuevo Mundo Mundos Nuevos [En línea]. https://doi.org/10.4000/nuevomundo.71626

FAO. Organización de las Naciones Unidas para la Alimentación y la Agricultura. (2013). Alianzas público-privadas para el desarrollo de agronegocios. Informe de país: Chile. Estudios de casos de países - América Latina. https://bit.ly/3J8SL3C

Fundación Codespa. (2013). Alianzas público-privadas para el desarrollo. Modelos para llevar a la práctica la innovación social empresarial. El autor. https://bit.ly/3suEFnr

Gabor, D. (1970). Innovations: Scientific, Technological and Social. Oxford University Press.

Garvey, W. D., \& Griffith, B. C. (1966). Studies of social innovations in scientific communication in psychology. American Psychologist, 21(11), 1019-1036. https://doi.org/10.1037/h0024053

Gordon, A., Becerra, L. D., \& Fressoli, M. (2017). Potentialities and constraints in the relation between social innovation and public policies: some lessons from South America. Ecology and Society, 22(4), 2. https://doi.org/10.5751/ES-09493-220402

Hernández-Ascanio, J., Tirado Valencia, P. y Ariza-Montes, A. (2016). El concepto de innovación social: ambitos, definiciones y alcances teóricos. CIRIEC-España, 88, 165-199. https://bit.ly/3qmAd7A

Hetland, G. \& Evans, P. (2016). From Illustrating Problems to Offering Solutions: Llatin America as a global source of social innovation. En M. Gutmann \& J. Lesser (Ed.), Global Latin America: Into the Twenty-First Century (pp. 72-88). University of California Press. https://doi.org/10.1525/9780520965942-011

Howaldt, J., Domanski, D. \& Kaletka, C. (2016). Social innovation: towards a new innovation paradigm. Mackenzie Management Review, 17(6), Special Edition, 20-44. https://doi.org/10.1590/167869712016/administracao.v17n6p20-44 
Jessop, B., Moulaert, F., Hulgård, L., \& Hamdouch, A. (2013). Social innovation research: a new stage in innovation analysis? En F. Moulaert, D. MacCallum, A. Mehmood, A., \& A. Hamdouch (Eds.), The International Handbook on Social Innovation. Collective Action, Social Learning and Transdisciplinary Research (pp. 110-130). Edward Elgar.

Martínez, R., Cruz, H., Blanco, I. y Salazar, Y. (2019). La innovación social, ¿prácticas para producir autonomía, empoderamiento y nueva institucionalidad? Revista Internacional de Sociología, 77(2), e126. https://doi.org/10.3989/ris.2019.77.2.17.022

Monge, N. \& Allamand, A. (2016). Innovación Social y valor compartido: el cambio de paradigma de la intervención social empresarial. En D. Domanski, N. Monge, G. Quitiaquez, \& Rocha, D. (Eds.), Innovación Social en Latinoamérica (pp. 71-92). Corporación Universitaria Minuto de Dios, Parque Científico de Innovación Social.

Morande, F. (2016). A casi tres décadas del Consenso de Washington ¿Cuál es su legado en América Latina?. Estudios Internacionales [Santiago, en línea], 48(185), 31-58. http://dx.doi.org/10.5354/0719-3769.2016.44553

Moreno-Brid, J.C., Pérez, E., \& Ruiz, P. (2004). El Consenso de Washington: aciertos, yerros y omisiones. Perfiles Latinoamericanos, 25, 149-168. https://bit.ly/3eb0lqH

Moulaert, F., MacCallum, D., \& Hillier, J. (2013). Social innovation: intuition, precept, concept, theory and practice. En F. Moulaert, D. MacCallum, A. Mehmood, A., \& A. Hamdouch (Eds.), The International Handbook on Social Innovation. Collective Action, Social Learning and Transdisciplinary Research (pp. 13-24). Edward Elgar.

Moulaert, F., MacCallum, D., Mehmood, A., \& Hamdouch, A. (2013). General introduction: the return of social innovation as a scientific concept and a social practice. En F. Moulaert, D. MacCallum, A. Mehmood, A., \& A. Hamdouch (Eds.), The International Handbook on Social Innovation. Collective Action, Social Learning and Transdisciplinary Research (pp. 1-6). Edward Elgar.

Murray, R., Caulier-Grice, J. y Mulgan, G. (2010). The Open Book of Social Innovation. The Young Foundation; Nesta. https://bit.ly/32f7Tfa

Naciones Unidas. (1995, marzo 6-12). Informe de la Cumbre Mundial sobre Desarrollo Social. https://bit.ly/3sovd4F

Ocampo, J.A. (2008). El auge económico latinoamericano. Revista de Ciencia Política, 28(1), 7-33. http://dx.doi.org/10.4067/S0718-090X2008000100002

Ocampo, J.A. (2014). La crisis latinoamericana de la luz de la historia. En J.A. Ocampo, B. Stallings, I. Bustillo, H. Belloso, \& R. Frenkel, La crisis latinoamericana de la deuda desde la perspectiva histórica. Naciones Unidas.

Özbağ, G.K, Esen, M. y Esen, D. (2019). Bibliometric analysis of studies on social innovation. International Journal of Contemporary Economics and Administrative Sciences, 9(1), 22-45. https://doi.org/10.5281/zenodo.3262221

Pacheco, A.S.V., Santos, M.J. \& Da Silva, K.V. (2018). Social innovation: what do we know and do not know about it. International Journal of Innovation and Learning, 24(3), 301-326. https://bit.ly/3mnq9tk 
Parada, J., Ganga, F., \& Rivera, Y. (2017). Estado del arte de la innovación social: una mirada a la perspectiva de Europa y Latinoamérica. Opción, 33(82), 563-587. https://bit.ly/3smfNxR

Rey de Marulanda, N. \& Tancredi, F. (2010). De la innovación social a la política pública. Naciones Unidas.

Rodríguez, A. \& Alvarado, H. (2008). Claves de la innovación social en América latina y el Caribe. Naciones Unidas.

Rodríguez, L. (2020). El tiempo social de las alianzas público privadas transnacionales en América Latina: un estudio de caso (2002-2010). Izquierdas, 49, 104-122. http://dx.doi.org/10.4067/S0718-50492020000100207

Taylor, J.B. (1970). Introducing Social Innovation. The Journal of Applied Behavioral Science, 6(1), 69-77. https://doi.org/10.1177/002188637000600104

Van der Have, R.P. \& Rubalcaba, L. (2016). Social innovation research: An emerging area of innovation studies? Research Policy, 45(9), 1923-1935. https://doi.org/10.1016/j.respol.2016.06.010

Weerakoon, C., McMurray, A., Rametse, N., \& Douglas, H. (2016, February 10-12). Social Innovation: A Preliminary Bibliometric Ana'ysis. Procesdiricis of the 3rd Social Innovation and Entreprene:urship Conference (SIERC), Aurkland, New Zealand (pp. 345-360). httrns://hit.lv/3s×2HOK

Westiey, F. (2013). Social innovacion and Resilience: How One Enhances the Other. Stanford Social

Copyright de la editorial: @2021 Luz Andrea Cote \& Emilio Ricci.

Este es un artículo de acceso abierto, bajo licencia Creative Commons BY 4.0. Innovation Review (Summer), 6-8. https://doi.org/10.48558/x1bz-ak47

Westley, F. \& Antadze, N. (2010). Making a Difference. Strategies for Scaling Social Innovation for Greater Impact. The Innovation Journal: The Public Sector Innovation Journal, 15(2), 1-19. https://bit.ly/3smgnM3

\section{Para citar este artículo bajo APA 7}

DOI

Williamson, J. (2004, enero 13). The Washington Consensus as policy prescription for development. Institute for International Economics, Banco Mundial [serie Practitioners of Development, Distribuida por el Banco Mundial]. https://bit.ly/3FkeXFw 